\title{
Grounding line transient response in marine ice sheet models
}

\author{
A. S. Drouet ${ }^{1}$, D. Docquier ${ }^{2}$, G. Durand ${ }^{1}$, R. Hindmarsh ${ }^{3}$, F. Pattyn ${ }^{2}$, O. Gagliardini ${ }^{1,4}$, and T. Zwinger \\ ${ }^{1}$ UJF- Grenoble 1/CNRS, Laboratoire de Glaciologie et Géophysique de l'Environnement (LGGE), UMR 5183, \\ Grenoble, 38041, France \\ ${ }^{2}$ Laboratoire de Glaciologie, Université Libre de Bruxelles, CP160/03, Av F. Roosevelt 50, 1050 Brussels, Belgium \\ ${ }^{3}$ British Antarctic Survey, Natural Environment Research Council, Madingley Road, Cambridge CB3 OET, UK \\ ${ }^{4}$ Institut Universitaire de France, Paris, France \\ ${ }^{5}$ CSC-IT Center for Science Ltd., Espoo, Finland
}

Correspondence to: G. Durand (durand@lgge.obs.ujf-grenoble.fr)

Received: 24 July 2012 - Published in The Cryosphere Discuss.: 18 September 2012

Revised: 11 January 2013 - Accepted: 14 January 2013 - Published: 1 March 2013

\begin{abstract}
Marine ice-sheet stability is mostly controlled by the dynamics of the grounding line, i.e. the junction between the grounded ice sheet and the floating ice shelf. Grounding line migration has been investigated within the framework of MISMIP (Marine Ice Sheet Model Intercomparison Project), which mainly aimed at investigating steady state solutions. Here we focus on transient behaviour, executing short-term simulations $(200 \mathrm{yr})$ of a steady ice sheet perturbed by the release of the buttressing restraint exerted by the ice shelf on the grounded ice upstream. The transient grounding line behaviour of four different flowline ice-sheet models has been compared. The models differ in the physics implemented (full Stokes and shallow shelf approximation), the numerical approach, as well as the grounding line treatment. Their overall response to the loss of buttressing is found to be broadly consistent in terms of grounding line position, rate of surface elevation change and surface velocity. However, still small differences appear for these latter variables, and they can lead to large discrepancies ( $>100 \%)$ observed in terms of ice sheet contribution to sea level when cumulated over time. Despite the recent important improvements of marine ice-sheet models in their ability to compute steady state configurations, our results question the capacity of these models to compute short-term reliable sea-level rise projections.
\end{abstract}

\section{Introduction}

A range of observational methodologies have shown that significant loss of Antarctic ice mass has occurred over the past decade (Wingham et al., 2006; Rignot et al., 2008, 2011; Velicogna, 2009; Pritchard et al., 2012). Increased basal melt of ice shelves appears to be the primary control on Antarctic ice sheet loss. Its resultant thinning induces a reduction of the buttressing force, i.e. the mechanical effect of the ice shelf on the state of stress of grounded ice, which leads to an acceleration of outlet glaciers (Rignot et al., 2008; Pritchard et al., 2012). The dynamical response of the grounding line (GL), where ice loses contact with bed and, downstream, begins to float over the ocean, is an essential control on the mass balance of a marine ice sheet. In particular, a rigorous mathematical description of the long-standing hypothesis of marine ice-sheet instability (Weertman, 1974) has been recently given by Schoof (2007) for a flowline type ice sheet without buttressing.

While observations are crucial in diagnosing the state of balance of an ice sheet, extrapolation of current trends is a limited technique in predicting ice-sheet future behaviour. Ice sheet models are therefore the central tool in forecasting the evolution of ice masses and, more particularly, their future contribution to the ongoing sea-level rise (SLR). A large suite of ice-sheet models has been developed in recent years. Increasing complexity has been regularly added, enabling progressive improvements from 1-D flowline models based on shallow-ice approximations to full numerical solutions of the Stokes equations for an actual 3-D geometry (Morlighem 
et al., 2010; Gillet-Chaulet and Durand, 2010; Larour et al., 2012; Gillet-Chaulet et al., 2012). However, implementing GL migration in ice-flow models still represents a challenge to be faced by the community of ice-sheet modellers (Vieli and Payne, 2005; Pattyn et al., 2012a,b).

As mentioned above, Schoof (2007) developed a boundary-layer theory establishing the relation between ice flux and ice thickness at the GL, which can be implemented as a boundary condition in ice-flow models. The boundary layer is a zone of acceleration, generally a few tens of kilometres in extent (Hindmarsh, 2006; Schoof, 2007) for highslip cases such as we consider, where the stress regime adjusts from being shear-dominated to extension-dominated. This theoretical development demonstrated the uniqueness of steady solutions of marine ice sheets resting on a downward sloping bedrock and their unstable behaviour on an upward sloping region. Based on the Schoof (2007) results, an intercomparison effort compared the behaviour of the GL evolution of 26 different models on a flowline, as part of the Marine Ice-Sheet Model Intercomparison Project (MISMIP, Pattyn et al., 2012a), which was essentially designed to compare models with the semi-analytical solution proposed by Schoof (2007). However, Schoof's flux formula is derived on the assumption of near-steady-state, and its ability to represent transient behaviour has not been fully investigated. This issue was briefly touched upon during the MISMIP experiments (Pattyn et al., 2012a), but it was not the primary focus of investigation.

The MISMIP experiments showed a broad range of behaviour of numerical implementations in response to an instantaneous global change of the ice rheology, with some quantitative consistency between different numerical formulations. The MISMIP experiments highlighted, along with Schoof's studies, the importance of obtaining high accuracy in the numerical solution in the boundary layer near the GL, which in practice means the use of high resolution or high accuracy methods, which has the consequence that the numerical approach used is of significant issue.

Short-term predictions of rapid change in the Antarctic Ice Sheet necessarily involve transient processes, and the ability of marine ice-sheet models to represent these requires quantification. Therefore, we conduct a model intercomparison dealing with rapid change in order to evaluate the transient behaviour of different models. A particular aim is to investigate the divergence of ice-sheet models from the Schoof (2007) solution during these very short time scale processes. Furthermore, owing to the use of different physical approximations and numerical approaches, we expect that the same experiment carried out with different ice sheet models may give different results. Therefore, another aim of this study is to quantify these differences and understand their origin.

In contrast to the original MISMIP experiment, here we choose to investigate the physically more reasonable transient forcing of a decrease in ice-shelf buttressing. This is implemented by means of a flowline model with a grounded part and a floating ice shelf. As is common with previous studies (Nick et al., 2009; Price et al., 2011; Williams et al., 2012), buttressing is implemented by varying the force applied at the calving front (downstream end) of the ice shelf. This is not an exact representation of how ice shelves generate back-pressure (Gagliardini et al., 2010), but since our primary focus is on how a release in back-pressure at the GL forces GL motion, this is sufficient for our purposes.

A recent study (Williams et al., 2012) has shown that the shallow-ice approximation, besides being invalid at short wavelength, is also invalid at sub-decadal to decadal forcing frequencies. This highlights the need to consider the nature of the mechanical model deployed in transient studies. Icesheet modelling has previously mainly been achievable with vertically-integrated mechanical representations of the appropriate governing Stokes equations. With recent advances, one of the models deployed solves the Stokes equations, while the others solve the vertically-integrated shallow-shelf approximation (SSA) (Morland, 1987; Mac Ayeal, 1992). The four models differ thus in the mechanical model as well as in the numerical approach used. They are briefly outlined here, with more details to follow below.

The first one is the finite-element full Stokes Elmer/Ice model, denoted $F S-A G$ (Full Stokes-Adaptive Grid), first presented in Durand et al. (2009b) (http://elmerice.elmerfem. org). In this application, an adaptive grid refinement is used. This model is computationally two-dimensional in this flowline representation. The three remaining models solve the SSA, and are therefore vertically integrated and thus computationally one-dimensional. SSA-FG (SSA-Fixed Grid) and $S S A-H-F G$ (SSA-Heuristic-Fixed Grid) use a fixed grid with a resolution of $50 \mathrm{~m}$ and $10 \mathrm{~km}$, respectively. The GL migration of $S S A-H-F G$ is computed according to the Pollard and DeConto (2009) heuristic rule that implements the Schoof (2007) boundary condition (Docquier et al., 2011). The last model solves the SSA equations using pseudospectral method (Fornberg, 1996; Hindmarsh, 2012) on a moving grid, and will be denoted SSA-PSMG (SSA-PseudoSpectral Moving Grid). For this model, grounded ice and floating ice shelf are solved on two coupled domains, with continuity of stress and velocity across the grounding line guaranteed. The first two models approach the problem of modelling the flow in the boundary layer by increased resolution, the third model uses a coarse resolution and a heuristic rule at the GL, and the last model addresses this issue by using high-accuracy spectral methods and explicit groundingline motion formula (Hindmarsh and LeMeur, 2001). All models have successfully participated in the MISMIP benchmark (Pattyn et al., 2012a), exhibiting unique stable positions on downward sloping beds, unstable GL positions on retrograde slopes and related hysteresis behaviour over an undulated bedrock.

Details and numerical characteristics of the four models are summarised in Table 1. In Sect. 2, specificities of the models are further described. The setup of the proposed 
Table 1. Summary table of model characteristics: LGGE/CSC stands for Laboratoire de l'Environnement, CSC for CSC-IT Center for Science, ULB for Université Libre de Bruxelles, and BAS for British Antarctic Survey.

\begin{tabular}{|c|c|c|c|c|}
\hline & $F S-A G$ & $S S A-H-F G$ & $S S A-F G$ & $S S A-P S M G$ \\
\hline Affiliation & $\begin{array}{l}\text { LGGE/CSC } \\
\text { (Durand et al., 2009a) }\end{array}$ & $\begin{array}{l}\text { ULB } \\
\text { (Docquier et al., 2011) }\end{array}$ & ULB & BAS \\
\hline Physics & full Stokes & SSA & SSA & SSA \\
\hline Numerics & finite element & finite difference & finite difference & pseudo-spectral \\
\hline Vertically integrated & no & yes & yes & yes \\
\hline Grid & adaptive & fixed and staggered & fixed and staggered & moving \\
\hline Resolution & GL: $50 \mathrm{~m}$; divide: $10 \mathrm{~km}$ & $10 \mathrm{~km}$ & $50 \mathrm{~m}$ & $3 \mathrm{~km}$ \\
\hline Time step & $0.1 \mathrm{yr}$ & $0.1 \mathrm{yr}$ & $0.1 \mathrm{yr}$ & $1 \mathrm{yr}$ \\
\hline GL & contact problem & $\begin{array}{l}\text { heuristic rule } \\
\text { (Pollard and DeConto, 2009) }\end{array}$ & flotation & margin tracking \\
\hline
\end{tabular}

experiments is outlined in Sect. 3 and corresponding results are discussed in Sect. 4 before we conclude in Sect. 5 .

\section{Model description}

\subsection{Governing equations}

The problem consists of solving a gravity-driven flow of incompressible and isothermal ice sliding over a rigid bedrock noted $b(x)$. The ice is considered as a non-linear viscous material, following the behaviour of the Glen's flow law (Glen, 1955):

$\boldsymbol{\tau}=2 \eta \mathbf{D}$,

where $\boldsymbol{\tau}$ is the deviatoric stress tensor, $\mathbf{D}$ is the strain rate tensor defined as $D_{i j}=\left(\partial_{j} u_{i}+\partial_{i} u_{j}\right) / 2$, and $\boldsymbol{u}=(u, w)$ is the velocity vector. The effective viscosity $\eta$ is defined as follows:

$\eta=\frac{A^{-1 / n}}{2} D_{e}^{(1-n) / n}$

where $A$ and $n$ are the Glen's law parameter and flow law exponent, respectively, and $D_{e}$ is the strain-rate invariant defined as $D_{e}^{2}=2 D_{i j} D_{i j}$.

The ice flow is computed by solving the Stokes problem, expressed by the mass conservation equation in the case of incompressibility:

$\operatorname{tr}(\mathbf{D})=\operatorname{div}(\boldsymbol{u})=0$,

and by solving the linear momentum balance equation:

$\operatorname{div}(\boldsymbol{\sigma})+\rho_{i} \boldsymbol{g}=0$,

where $(\boldsymbol{\sigma})=\boldsymbol{\tau}-p \boldsymbol{I}$ is the Cauchy stress tensor with $p=$ $-\operatorname{tr} \sigma / 3$ the isotropic pressure, $\rho_{i}$ the ice density and $\boldsymbol{g}$ the gravity vector.

Both the upper ice/atmosphere interface $z=z_{\mathrm{s}}(x, t)$ and the lower ice/bedrock or ocean interface $z=z_{\mathrm{b}}(x, t)$ are allowed to evolve following an advection equation:

$\frac{\partial z_{i}}{\partial t}+u_{i} \frac{\partial z_{i}}{\partial x}-w_{i}=a_{i} \quad i=s, b$,

where $\left(u_{i}, w_{i}\right)$ is the surface velocity $(i=s)$ or the basal velocity $(i=b)$. For this application, the mass flux at the surface (i.e. surface mass balance) is constant and uniform $\left(a_{\mathrm{s}}(x, t)=a_{\mathrm{s}}\right.$, see Table 2$)$ and $a_{\mathrm{b}}=0$.

\subsection{Boundary conditions}

The geometry is restricted to a two-dimensional flowline along the $\mathrm{x}$-direction and the $\mathrm{z}$-axis is the vertically upward direction. The upstream boundary of the domain $x=0$ is taken to be a symmetry axis (ice divide), where we impose the horizontal velocity $u(x=0)=0$. The downstream boundary, $x=x_{\mathrm{f}}$, corresponds to the calving front. The position of the calving front $x_{\mathrm{f}}$ is fixed, and the GL position $x_{\mathrm{g}}$ is delimited by $0 \leq x_{\mathrm{g}} \leq x_{\mathrm{f}}$. In what follows, we assume a constant sea level, set to $z=0$.

The upper ice surface $z=z_{\mathrm{s}}(x, t)$ is in contact with the atmosphere, where pressure is negligible with respect to involved stresses inside the ice body. This is a stress-free surface, implying the following condition:

$\left.\boldsymbol{\sigma} \cdot \boldsymbol{n}\right|_{z_{\mathrm{s}}}=0$

where $\boldsymbol{n}$ is the outward pointing unit normal vector.

The lower surface $z=z_{\mathrm{b}}(x, t)$ is either in contact with the bedrock or with the ocean, and two different boundary conditions will be applied for the Stokes problem on these two different interfaces, defined as follows:

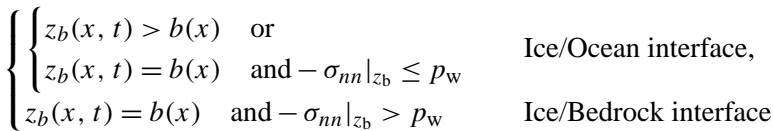

In Eq. (7), the water pressure $p_{\mathrm{w}}=p_{\mathrm{w}}(z, t)$ is defined as follows:

$p_{\mathrm{w}}(z, t)=\left\{\begin{array}{lll}-\rho_{\mathrm{w}} g z & \text { if } & z \leq 0 \\ 0 & \text { if } & z>0\end{array}\right.$ 
Table 2. Parameters of initial steady state.

\begin{tabular}{|c|c|c|c|}
\hline Parameter & Description & Value & Unit \\
\hline$b$ & Bed elevation & $-x / 1000$ & $\mathrm{~m}$ \\
\hline$\rho_{i}$ & Ice density & 900 & $\mathrm{~kg} \mathrm{~m}^{-3}$ \\
\hline$\rho_{\mathrm{W}}$ & Water density & 1000 & $\mathrm{~kg} \mathrm{~m}^{-3}$ \\
\hline$g$ & $\begin{array}{l}\text { Gravitational } \\
\text { acceleration }\end{array}$ & 9.8 & $\mathrm{~m} \mathrm{~s}^{-2}$ \\
\hline$A$ & $\begin{array}{l}\text { Glen's law } \\
\text { coefficient }\end{array}$ & $1.5 \times 10^{-25}$ & $\mathrm{~Pa}^{-3} \mathrm{~s}^{-1}$ \\
\hline$n$ & $\begin{array}{l}\text { Glen's law } \\
\text { exponent }\end{array}$ & 3 & \\
\hline C & $\begin{array}{l}\text { Basal friction } \\
\text { parameter }\end{array}$ & $10^{6}$ & $\mathrm{Pam}^{-1 / 3} \mathrm{~s}^{1 / 3}$ \\
\hline$m$ & $\begin{array}{l}\text { Basal friction } \\
\text { exponent }\end{array}$ & $1 / 3$ & \\
\hline$a_{\mathrm{s}}$ & $\begin{array}{l}\text { Accumulation } \\
\text { rate }\end{array}$ & 0.3 & $\mathrm{ma}^{-1}$ \\
\hline$C_{F}$ & $\begin{array}{l}\text { Buttressing } \\
\text { parameter }\end{array}$ & 0.4 & \\
\hline
\end{tabular}

where $\rho_{\mathrm{w}}$ is the water density.

Where the ice is in contact with the ocean (first condition in Eq. 7), the following Neumann boundary condition applies for the Stokes equations:

$\boldsymbol{\sigma} \cdot \boldsymbol{n}=-p_{\mathrm{w}} \boldsymbol{n}$.

Where the ice is in contact with the bedrock (second condition in Eq. 7), a no-penetration condition is imposed as well as a friction law, such as

$\boldsymbol{u} \cdot \boldsymbol{n}=0$,

$\tau_{\mathrm{b}}=\left.\boldsymbol{t} \cdot(\boldsymbol{\sigma} \cdot \boldsymbol{n})\right|_{\mathrm{b}}=C u_{\mathrm{b}}^{m}$,

where $\tau_{\mathrm{b}}$ is the tangential component of the traction, $t$ is the tangent vector to the bedrock, $u_{\mathrm{b}}$ is the sliding velocity, $C$ is the friction parameter and $m$ is the friction law exponent (see Table 2 for the adopted values).

\subsection{Shallow shelf/shelfy stream approximation (SSA)}

As mentioned previously, three of the four models use the shallow shelf approximation (SSA), which is a vertically integrated approximation of the Stokes Eqs. (3) and (4). The horizontal velocity $u(x)$ is obtained by solving the following equations (Morland, 1987; Mac Ayeal, 1992):

$\begin{cases}2 \frac{\partial\left(h \tau_{x x}\right)}{\partial x}-C u^{m}=\rho_{i} g h \frac{\partial z_{\mathrm{s}}}{\partial x} & 0 \leq x \leq x_{\mathrm{g}}, \text { for the grounded part, } \\ 2 \frac{\partial\left(h \tau_{x x}\right)}{\partial x}=\gamma h \frac{\partial h}{\partial x} & x_{\mathrm{g}}<x \leq x_{\mathrm{f}}, \text { for the floating part }\end{cases}$

where $h=h(x)$ is the ice thickness, $\tau_{x x}=2 \eta \partial_{x} u$ is the longitudinal deviatoric stress and $u$ is the horizontal velocity in the flow direction. The effective viscosity, $\eta$, is computed as
Table 3. GL position for the intial steady state $\left(C_{F}=0.4\right)$ and for the different perturbations for each model after $200 \mathrm{yr}$. The difference between the initial steady state and the perturbed state is given in brackets. All values are in $\mathrm{km}$.

\begin{tabular}{lrrrr}
\hline & $F S-A G$ & $S S A-F G$ & $S S A-H-F G$ & $S S A-P S M G$ \\
\hline$C_{F}=0.4$ & 540.5 & 551.8 & 554.1 & 556.1 \\
$C_{F}=0.5$ & $523.8(16.7)$ & $534.7(17.1)$ & $530.4(23.8)$ & $539.2(16.9)$ \\
$C_{F}=0.8$ & $482.0(58.5)$ & $488.5(63.3)$ & $474.8(79.3)$ & $495.2(60.9)$ \\
$C_{F}=1$ & $463.7(76.8)$ & $468.9(82.9)$ & $454.3(99.8)$ & $476.8(79.3)$ \\
\hline
\end{tabular}

in Eq. (2), where $D_{e} \approx \partial_{x} u$. The parameter $\gamma$ is defined as

$\gamma=\rho_{i} g\left(1-\frac{\rho_{i}}{\rho_{\mathrm{w}}}\right)$

According to the SSA approximation, ice deformation is dominated by membrane stresses and vertical shear within the ice is neglected. For the SSA model, the only boundary condition is $u(x=0)=0$ at the ice divide, whereas the boundary condition at the lower surface is already implicitly included in the set of Eq. (11) and the boundary condition at the calving front is defined in Sect. 2.5.

The lower surface $z_{\mathrm{b}}$ is determined from the nopenetration condition and the floating condition:

$\begin{cases}z_{\mathrm{b}}(x, t)=b(x) & \text { for } x \leq x_{\mathrm{g}}, \\ z_{\mathrm{b}}(x, t)=-h \rho_{i} / \rho_{\mathrm{w}}>b(x) & \text { for } x>x_{\mathrm{g}} .\end{cases}$

The upper surface $z_{\mathrm{s}}=z_{\mathrm{b}}+h$ is deduced from the vertically-integrated mass conservation equation, giving $h$ as follows:

$\frac{\partial h}{\partial t}+\frac{\partial(h u)}{\partial x}=a_{\mathrm{s}}$

\subsection{Grounding line treatment}

The implementation of GL treatment differs from one model to the other. In this section we define for each model the specificities regarding the treatment of the GL.

The FS-AG model solves the contact problem between the ice and the bedrock. During a time step, the contact condition (7) is tested at each node of the mesh and the bottom boundary conditions (9) or (10) are imposed accordingly. More details about this method and its implementation can be found in Durand et al. (2009a). The consistency of this GL implementation strongly depends on the grid resolution, and a grid size lower than $100 \mathrm{~m}$ is needed to obtain reliable results (Durand et al., 2009b). In order to reach this resolution while considering a reasonable number of mesh nodes, an adaptive mesh refinement around the GL is applied: the horizontal distribution of nodes is updated at every time step, such that finer elements are concentrated around the GL.

For the $S S A-F G$ model the grid points are kept fixed in time and the last grounded grid point is determined through the 
flotation criterion, i.e. by solving the following equation:

$F=h_{\mathrm{g}}+b\left(x_{\mathrm{g}}\right) \frac{\rho_{\mathrm{w}}}{\rho_{i}}=0$.

The GL position $x_{\mathrm{g}}$ is given with sub-grid precision between the last grounded grid point and the first floating point following the method proposed by Pattyn et al. (2006).

The GL position is also determined with sub-grid precision following Pattyn et al. (2006) for the $S S A-H-F G$, but while $S S A-F G$ uses the flotation criterion as a boundary condition at the GL, the $S S A-H-F G$ model makes use of an additional boundary condition based on the semi-analytical solution of Schoof (2007). The ice flux at the GL $q_{\mathrm{g}}$ is calculated as a function of ice thickness at the GL $h_{\mathrm{g}}$ :

$q_{\mathrm{g}}=\left(\frac{A \rho_{i} g \gamma^{n}}{4^{n} C}\right)^{\frac{1}{m+1}} \theta^{\frac{n}{m+1}} h_{\mathrm{g}}^{\frac{m+n+3}{m+1}}$,

and is used in a heuristic rule to enable GL migration (Pollard and DeConto, 2009). This parameterization allows relatively coarse resolutions to be used (10 km in this study) and gives steady-state results of GL position that are independent of the chosen resolution and agree well with the semi-analytical solution given by Schoof (2007) (Docquier et al., 2011). In Eq. (16), the coefficient $\theta$ accounts for buttressing and is defined as follows:

$\theta=\frac{\left.4 \tau_{x x}\right|_{x_{\mathrm{g}}}}{\gamma h_{\mathrm{g}}}$.

The numerical approach used by the pseudo-spectral SSA$P S M G$ model consists in explicitly calculating the rate of GL migration, $\dot{x}_{\mathrm{g}}$, according to the following explicit formula (Hindmarsh and LeMeur, 2001):

$\dot{x}_{\mathrm{g}}=-\frac{\partial_{t} F}{\partial_{x} F}$,

where $F$ is given by Eq. (15). At each time step, a new position is computed and the grid moves accordingly, so that the GL coincides exactly with a grid point (Hindmarsh, 1993). Moving grids have the ability to ensure that a grid point always coincides with the GL, allowing easy representation of gradients at this location, but are not always convenient to implement.

\subsection{Calving front boundary condition and the specification of buttressing}

The experiments we propose are driven by changes in the buttressing force. One approach could have consisted of applying lateral friction on the ice shelf following the method of Gagliardini et al. (2010), but the total buttressing force would then have been a function of the ice-shelf area and ice-shelf velocities, and therefore different for all models. In order to ensure the same buttressing force for all models, we follow the method proposed by Price et al. (2011), in which the inward force at the calving front is modified by a factor, noted $C_{F}$ in our study.

For vertically integrated models, the horizontal force acting on the calving front is entirely due to the hydrostatic water pressure and the longitudinal deviatoric stress at the front is given by MacAyeal et al. (1996):

$\left.\tau_{x x}\right|_{x_{\mathrm{f}}}=\frac{\gamma}{4} h_{f}$,

where $h_{\mathrm{f}}$ is the ice thickness at the calving front. In the case of the vertically integrated models $S S A-F G, S S A-H-F G$ and $S S A-P S M G$, a factor $C_{F}$ is then used to modify longitudinal deviatoric stress (19), which becomes the following:

$\left.\tau_{x x}\right|_{x_{f}}=C_{F} \frac{\gamma}{4} h_{\mathrm{f}}$.

A value of $C_{F}=1$ means that the longitudinal deviatoric stress at the calving front is opposed solely by water pressure, corresponding to no buttressing. Values less than one induce a lower extensional longitudinal deviatoric stress at the front, simulating the effect of buttressing. Note that this procedure implies an additional force applied at the calving front; this results in a varying contribution of the butressing to the stress as the ice thickens upstream.

Moreover, for $S S A-H-F G$, the buttressing parameter $C_{F}$ is by construction incorporated in the boundary condition at the GL. This boundary condition relates the ice flux $q_{\mathrm{g}}$ to the ice thickness $h_{\mathrm{g}}$ at the GL and includes the buttressing factor $\theta$ as defined by Eq. (17). From the SSA equations in the ice shelf, we derive (see Appendix A) the relation that links $\theta$ and $C_{F}$ through both the ice thickness at the GL $h_{\mathrm{g}}$ and the ice thickness at the calving front $h_{\mathrm{f}}$ :

$\theta=1-\left(1-C_{F}\right)\left(\frac{h_{\mathrm{f}}}{h_{\mathrm{g}}}\right)^{2}$.

The other two SSA models solve for the longitudinal variation of $\tau_{x x}$ in the ice shelf to compute the value at the GL.

For the $F S-A G$ model, the hydrostatic pressure $p_{\mathrm{w}}(z)$ is imposed along the ice column in contact with the sea, so that the longitudinal Cauchy stress is not uniform on this boundary. This non-uniform stress induces a bending of the ice shelf near the front. To avoid an increase of this bending when adding the buttressing, the stress condition at the front is modified by adding a uniform buttressing stress $p_{\mathrm{b}}$ :

$\left.\sigma_{x x}\right|_{x_{f}}(z, t)=p_{\mathrm{w}}(z)+p_{\mathrm{b}}(t)$

Using (Eqs. 22 and 20), and assuming the equality of the mean longitudinal Cauchy stress for both parameterisations, the buttressing stress to be applied at the front of the full Stokes model is obtained as a function of $C_{F}$ (see Appendix B), such as the following:

$p_{\mathrm{b}}=\frac{\rho_{\mathrm{w}} g z_{\mathrm{b}}^{2}}{2 \rho_{i} h_{\mathrm{f}}}\left(\rho_{\mathrm{w}}-\rho_{i}\right)\left(C_{F}-1\right)$. 


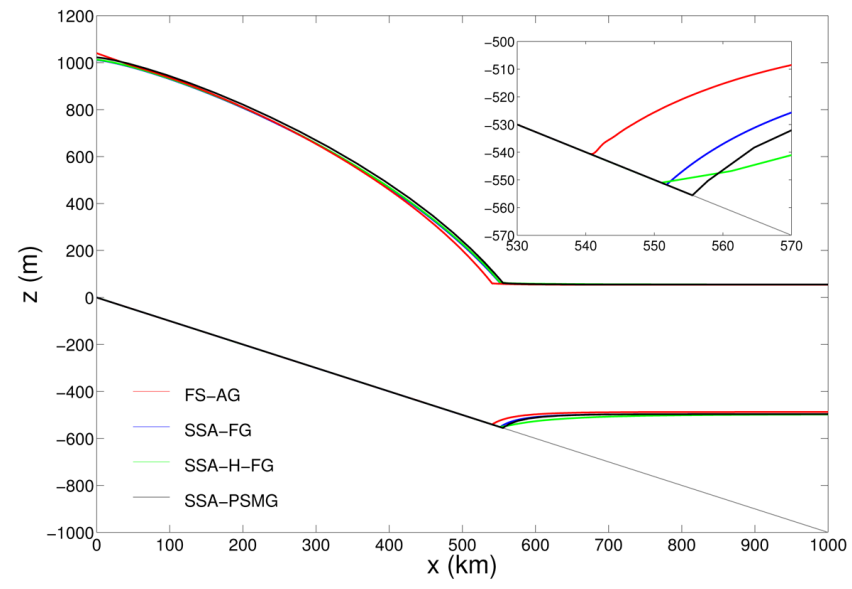

Fig. 1. Initial steady state geometry $\left(C_{F}=0.4\right)$ for all models. The inset emphasizes the differences in GL position. $S S A-H-F G$ lower surface has a quite different shape with respect to the three other models due to its coarser resolution $(10 \mathrm{~km})$.

Note that $p_{\mathrm{b}}$ has to be computed at each time step since it depends on the ice thickness at the front, which is not constant.

\section{Experimental setup}

We consider an ice sheet resting on a downward sloping bedrock, with the calving front fixed at $1000 \mathrm{~km}$, as shown in Fig. 1. The GL never advances as far as this in the experiments. The flow parameters summarised in Table 2 are used by each model in order to calculate a steady-state geometry. The steady state is obtained with a buttressed ice shelf $\left(C_{F}=0.4\right)$.

Computed steady surfaces are in good agreement between models, exhibiting only a slight difference in GL position of less than $20 \mathrm{~km}$ (see Fig. 1).

We chose the simpler, stable case of a forward slope for the simple reason that computing comparable initial starting conditions on the unstable reverse slope is a practical impossibility. GL retreat rates are governed by the water depth and the buttressing, and we chose values that were physically acceptable and also produced physically reasonable retreat rates.

Ice-sheet geometry is subsequently perturbed by a release of the initial buttressing force. This process, arising from increased melt of the ice shelf, appears to be responsible for the observed acceleration of Antarctic outlet glaciers (Wingham et al., 2006; Rignot et al., 2008; Pritchard et al., 2012).
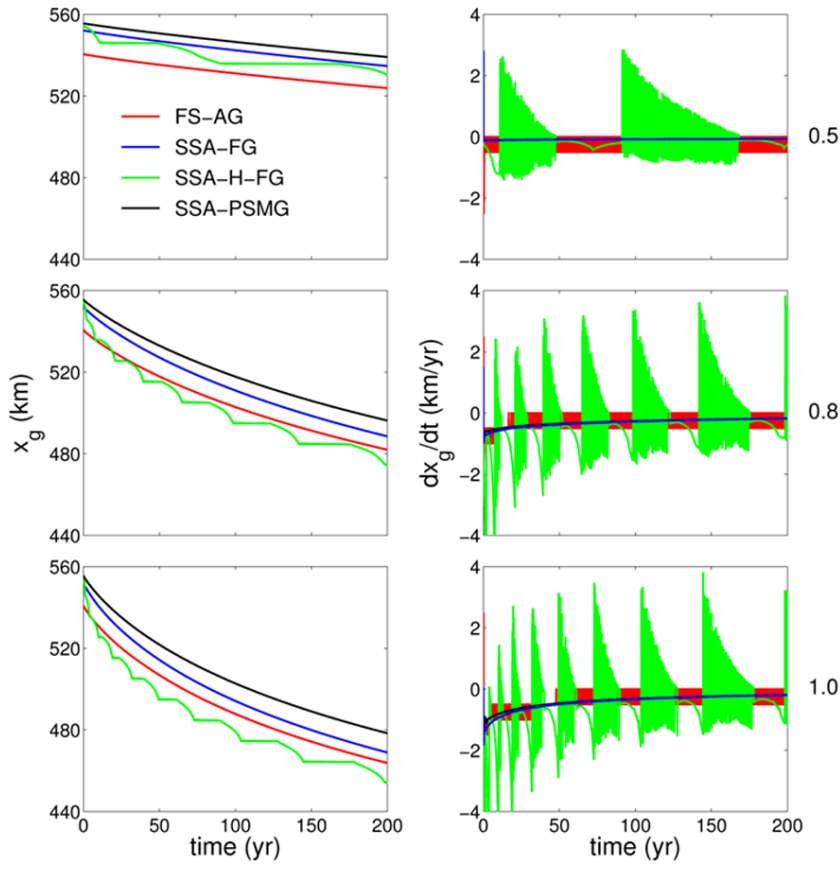

Fig. 2. Grounding line position $x_{\mathrm{g}}$ (left) and migration rate $\mathrm{d} x_{\mathrm{g}} / \mathrm{d} t$ (right) as a function of time for the four models and for the three buttressing values $\left(C_{F}=0.5\right.$ on the first line, $C_{F}=0.8$ on the second line and $C_{F}=1$ on the third line).

Starting from the steady geometries obtained with initial factor $C_{F}=0.4$, the buttressing force is decreased at $t=0$ (i.e. $C_{F}$ increases) and kept constant during the simulation. Since we focus on the transient behaviour, simulations are run for a short-term period of 200-yr. Three different amplitudes of the perturbation are investigated with corresponding modified values of $C_{F}=0.5,0.8$ and 1 .

\section{Results and discussion}

\subsection{Transient behaviour of direct observable variables on actual ice sheets}

We first evaluate the response of the various models regarding the variables that are currently observed over actual ice sheets, namely GL position (Fig. 2), surface elevation change (Fig. 3) and surface velocity (Fig. 4).

As expected, release of buttressing induces a GL retreat, and the greater the release, the larger the amount and rate of retreat (Gagliardini et al., 2010). Retreat can reach up to almost $100 \mathrm{~km}$ in $200 \mathrm{yr}$ following a complete loss of buttressing restraint $\left(C_{F}=1\right.$, see Fig. 2 and Table 3$)$. The different models show a similar trend regarding the temporal evolution of GL position (left panels in Fig. 2). However, owing to the various initial steady state profiles, the GL position differs between models. For the three perturbations, $S S A-H-F G$ 


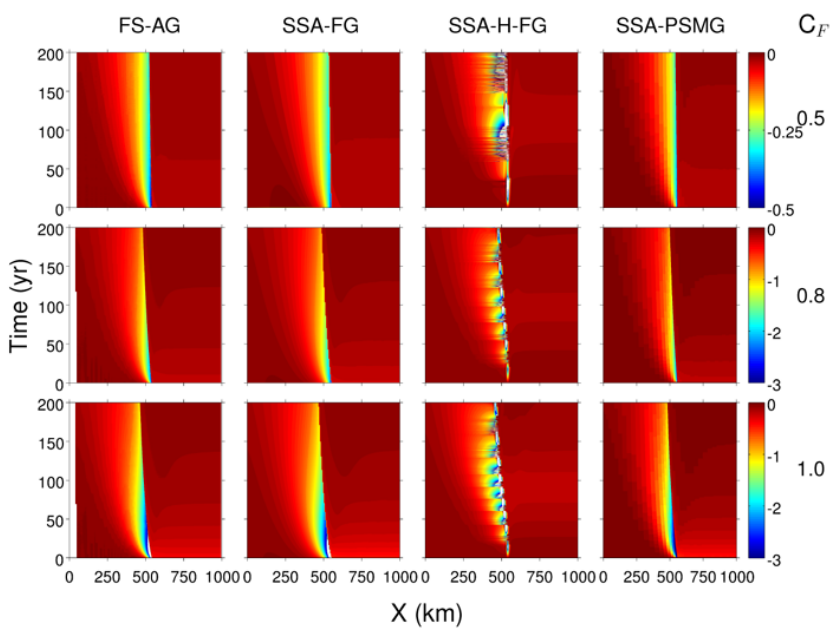

Fig. 3. Rate of surface elevation change $\left(\mathrm{m} \mathrm{yr}^{-1}\right)$ as a function of time and horizontal distance ( $x=0$ corresponds to the ice divide and $x_{\mathrm{f}}=1000 \mathrm{~km}$ is the calving front) for the three buttressing values (lines) and for the four models (columns).

shows the highest GL retreat compared to the initial position, followed by $S S A-F G$, then $S S A-P S M G$, and finally $F S-A G$ (Table 3).

The evolution of the GL position of $S S A-H-F G$ has a steplike behaviour due to the model grid size $(10 \mathrm{~km})$.

Rates of GL migration (right panels in Fig. 2) for SSA$P S M G$ and $S S A-F G$ exhibit a very similar pattern, i.e. a high retreat rate value in the beginning of the perturbation and then a convergence towards a zero-value. Moreover, the greater the perturbation (higher value of $C_{F}$ ), the larger the retreat rates in the beginning of the perturbation. The smooth decrease of the migration rate computed by $S S A-P S M G$ is due to the explicit way the GL migration is computed (see model description above). Because the $S S A-F G$ interpolates the GL position between the last grounded point and the first floating point (Pattyn et al., 2006), it also ensures a smooth description of GL migration rate. However, $F S-A G$ and $S S A-$ $H-F G$ show discontinuous GL migration rate induced by numerical artefacts as follows: both models give results that are affected by their grid size. The stepped patterns obtained with $F S-A G$ are due to high frequency oscillation between two successive nodes during GL migration: the GL retreats, then stays at the same position during one time step, then retreats, etc., so that the GL migration rate oscillates with an amplitude of $500 \mathrm{~m} \mathrm{a}^{-1}$ (i.e. grid size divided by time step). The numerical noise found in $S S A-H-F G$ is due to a combination of both the grid size effect and single-cell dithering, i.e. flipping back and forth between upstream and downstream grid points (Pollard and DeConto, 2012). As a general trend, the GL retreats by $10 \mathrm{~km}$ steps as a consequence of the model resolution (grid size effect). At some discrete GL positions (every $10 \mathrm{~km}$ ), the rate of GL migration varies significantly due to the heuristic rule used in the model (flux

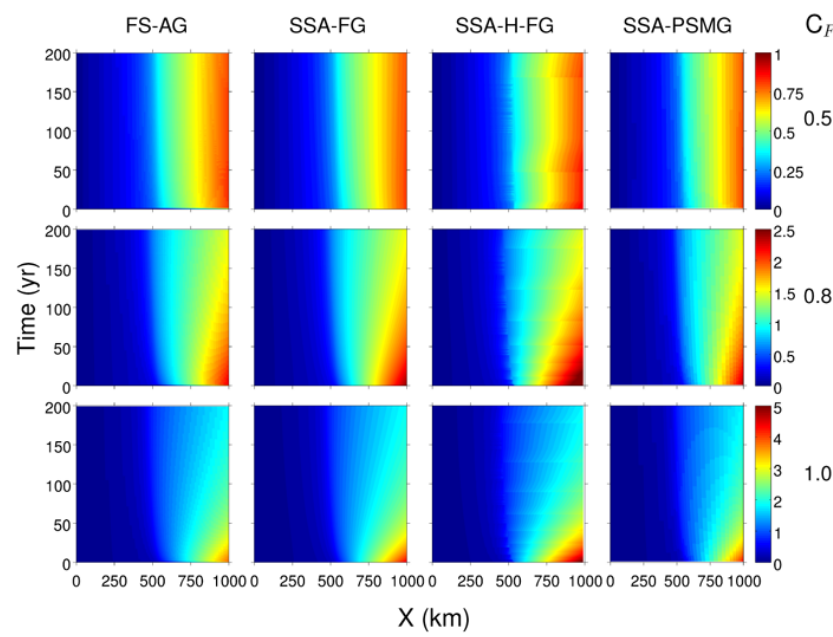

Fig. 4. Surface horizontal velocity $\left(\mathrm{km} \mathrm{yr}^{-1}\right)$ as a function of time and horizontal distance ( $x=0$ corresponds to the ice divide and $x_{\mathrm{f}}=1000 \mathrm{~km}$ is the calving front) for the three buttressing values (lines) and for the four models (columns).

imposed either upstream or downstream the GL), so that the GL slightly advances and retreats within the same grid cell (single-cell dithering). In summary, the GL retreats by $10 \mathrm{~km}$ (corresponding to the model resolution) and reaches a discrete position where it oscillates within the same grid cell, and then retreats before reaching another discrete position again, ad inf.

Rates of surface elevation change through time and distance from the ice divide are presented in Fig. 3 for the various models and perturbations. The horizontal surface velocity is similarly plotted (see Fig. 4). The largest perturbation $\left(C_{F}=1\right)$ exhibits rates of surface elevation change of a few meters per year in the beginning, with horizontal velocities above one kilometer per year. Together with GL migration rates of the order of a kilometer per year (Fig. 2), those are in general agreement with the obervation for currently recessing glaciers of West Antarctica, and Pine Island Glacier in particular (Rignot, 1998; Rignot et al., 2011). That confirms the relevance of the amplitude of the perturbations applied. Rates of surface elevation change are quite similar between the four models (Fig. 3). The highest thinning rates appear in the vicinity of the GL at the beginning of the perturbation. Similarly, the surface velocities steadily decrease during the simulation (Fig. 4). High frequency and small amplitude numerical noise in $F S-A G$ appear not to significantly affect the surface response. However, with $S S A-H-F G$ the high frequency and amplitude variabilities drastically affect the surface thinning rate and velocities over short time scales (i.e. about a decade).

We deliberately chose a low spatial resolution (uniform $10 \mathrm{~km}$ along the flowline) for the $S S A-H-F G$ model compared with other models. Indeed, in contrast to other approaches, such type of models produces consistent steady 
geometries at low spatial resolution (Docquier et al., 2011), which is the main motivation for applying such parameterizations in large-scale ice-sheet models. One can also note that ice-sheet models using a flux boundary condition at the grounding line with a similar resolution are currently used on centennial time scale to estimate Antarctic evolution (Bindschadler et al., 2012). For the numerical approaches of SSA$F G$ and $F S-A G$, they are known to fail to compute consistent steady geometries when too large resolution is used (Durand et al., 2009a). This would therefore make no sense to investigate their transient behaviours with too coarse a mesh. In our opinion, this justifies our choice of different mesh sizes from one model to the other according to the (i) capacity of each model to correctly compute steady states at a given resolution, and (ii) the mesh size currently used to predict ice-sheet short-term response. However, evaluating their performance with similar numerics on controlled experiments remains to be done. Increasing the resolution (down to $500 \mathrm{~m}$ ) for the $S S A-H-F G$ model allows removal of high frequency numerical artefacts, but the general trend of variables such as GL migration rate and surface elevation changes over $200 \mathrm{yr}$ does not depend on resolution (data not shown). Moreover, refining the grid size significantly increases its numerical cost, so that the major advantage of this model is lost, as well as its applicability to large-scale ice sheet models.

\subsection{Divergence from the boundary-layer solution}

Despite the numerical noise exhibited by $S S A-H-F G$ and $F S-$ $A G$ models, the evolution of the geometry during the simulations appears very similar for all four models. However, the boundary-layer theory implemented in the $S S A-H-F G$ model hypothesizes near-steady conditions and its ability to represent transients requires evaluation. In Fig. 5, the flux at the GL is plotted as a function of the instantaneous ice thickness at the GL for all models and simulations. By construction, $S S A-H-F G$ essentially follows the boundary-layer prescription. This can most clearly be seen for the case $C_{F}=1$ (see the bottom of Fig. 5) where the close correspondence of the curves of Schoof (2007) and SSA-H-FG is evident. This correspondence is not as clear for the other perturbations, since the $S S A-H-F G$ boundary condition for the flux now relies on a parameterization of $\theta$, which in turn depends on the quantity $h_{\mathrm{f}} / h_{\mathrm{g}}$ (see Eq. 21 ). Since this ratio varies in time, the steady-state condition of the Schoof condition is not fulfilled.

Interestingly, and despite their very different physical and numerical approaches, all the other models show very similar behaviour, with the boundary-layer theory result attained after some time. This is most obvious for the largest perturbation $\left(C_{F}=1\right)$ but also clearly visible for the weaker perturbations $\left(C_{F}=0.8\right.$ and 0.5$)$. However, during the highly transient phase, for a given ice thickness at the GL, the ice flux is substantially overestimated by the boundary layer theory, consequently overestimating the outflow during the period of $200 \mathrm{yr}$ period.

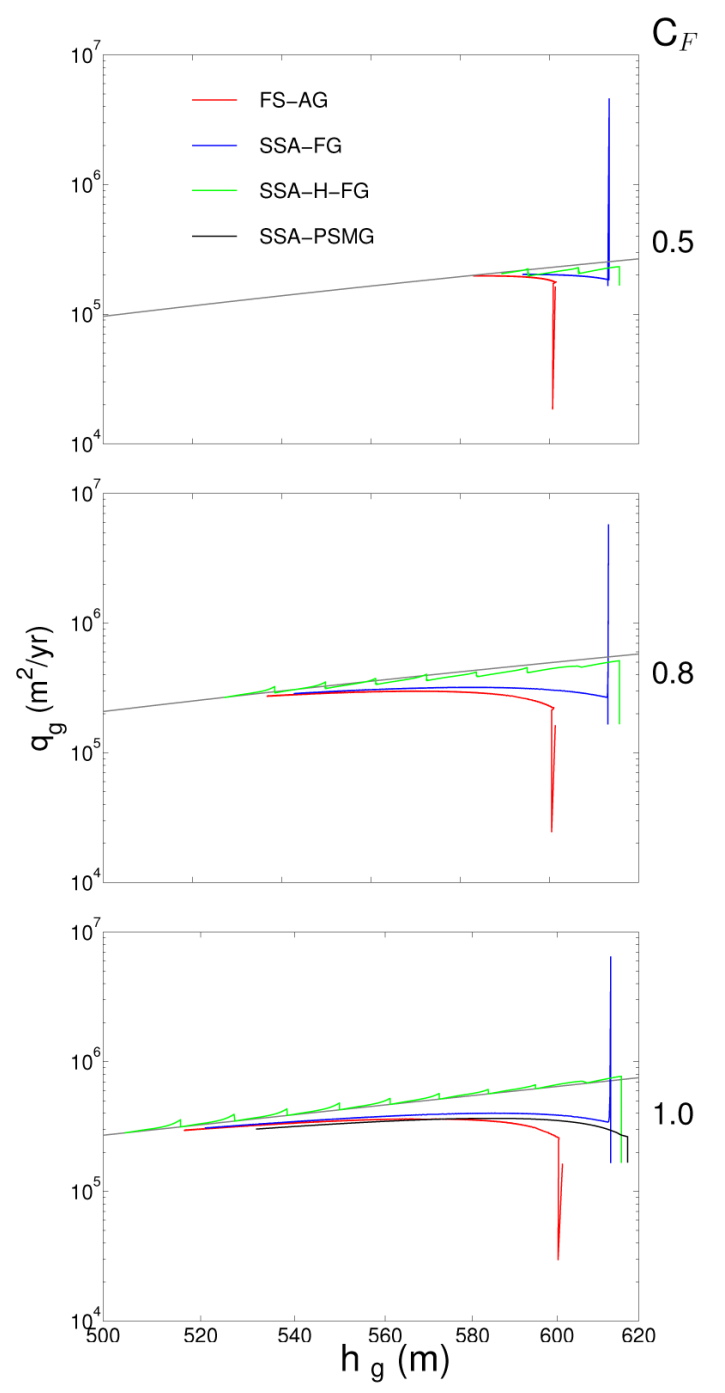

Fig. 5. GL ice flux $q_{\mathrm{g}}$ as a function of GL ice thickness $h_{\mathrm{g}}$ for the four models and for the three different buttressing values, compared with the Schoof (2007) solution (in grey).

\subsection{Changes in volume above flotation ( $\triangle$ VAF)}

From the perspective of projecting the future contribution of Antarctica to sea-level rise (SLR), the change in volume above flotation $(\triangle \mathrm{VAF}=\operatorname{VAF}(t)-\operatorname{VAF}(t=0))$ is certainly a pertinent variable to investigate. Indeed, plotting $\triangle \mathrm{VAF}$ (Fig. 6) has the advantage of integrating through time both the contribution coming from outflow at the GL and the consequence of grounding-line retreat in terms of ice release. In our case, this also allows the investigation of the spread in the transient behaviour of the various models in response to similar perturbations. We also plotted the evolution of $\triangle \mathrm{VAF}$ for each model relative to $\triangle \mathrm{VAF}$ computed by $F S-A G$, as it directly emphasizes the difference between models (Fig. 6). Choice of FS-AG model as a reference was arbitrary. 

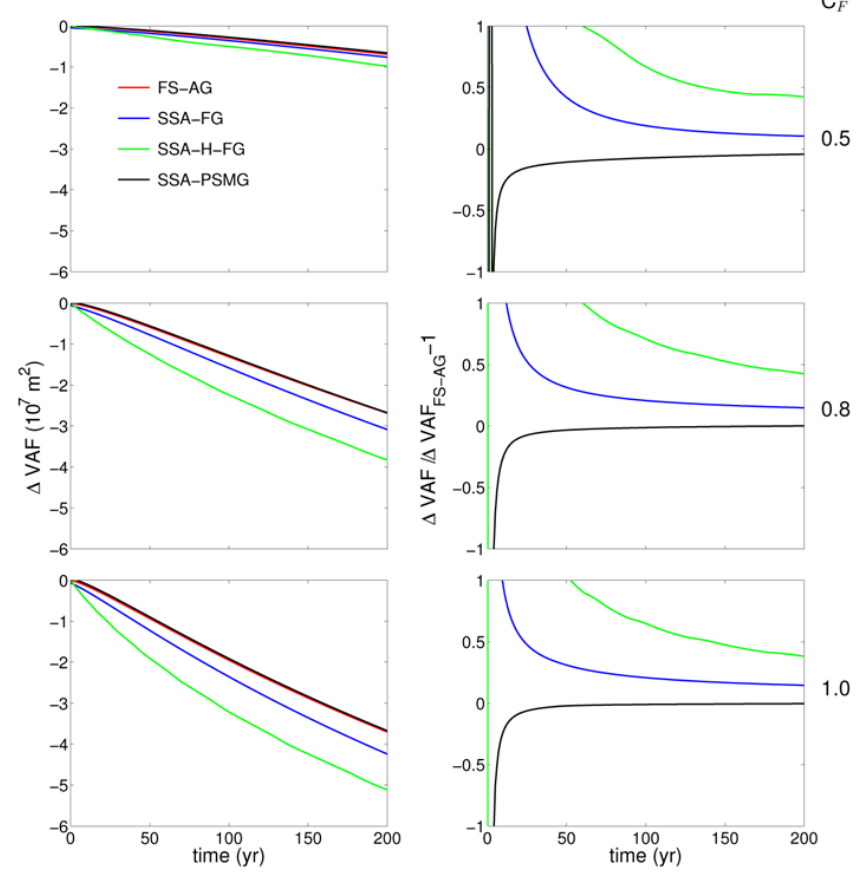

Fig. 6. Temporal evolution of the variation of Volume above Flotation ( $\triangle \mathrm{VAF}$ ) for each model (left) and, expressed relative to $F S-A G$ $\left(\triangle \mathrm{VAF}_{F S-A G}\right)$ for the three remaining models (right), for the three butressing values (lines).

As anticipated, $S S A-H-F G$ shows the greatest change in VAF compared with other models. Relative to $F S-A G, S S A-H-F G$ overestimates the contribution to SLR by more than $100 \%$ during the first $50 \mathrm{yr}$ of the simulation, which decreases to a $40 \%$ overestimation after $200 \mathrm{yr}$. $S S A-F G$ shows a similar pattern with a smaller overestimation (about $15 \%$ after $200 \mathrm{yr}$ ). On the other hand, SSA-PSMG briefly underestimates the change in VAF relative to $F S-A G$ at the beginning of the perturbation, but after $20 \mathrm{yr}$ the contribution of the models to SLR is remarkably similar to the one computed by $F S-A G$, with relative difference below $5 \%$. It seems striking that response in terms of relative $\triangle \mathrm{VAF}$ is extremely similar from one perturbation to the other, while the response of the models is highly modulated by the amplitude of the perturbation. This particularity may allow in the future to weight the response of a model according to the physics with which it is implemented.

This intercomparison strongly suggests that models prescribing flux at the GL according to the boundary-layer theory most probably overestimate ice discharge, with significant difference at the very beginning of the transient simulation. It also clearly shows that the rate of contribution to SLR significantly differs from one model to the other, even for a relatively simple and constrained experiment. When extrapolated to the current imbalance of the Antarctic ice sheet, this would have important consequences. According to Rignot et al. (2011), the Antarctic ice sheet drained about
$100 \mathrm{Gt} \mathrm{yr}^{-1}$ in 2000 with an increasing acceleration trend in mass loss of $14.5 \mathrm{Gt} \mathrm{yr}^{-1}$. Following that trend, the Antarctic ice sheet has contributed by $4.6 \mathrm{~mm}$ of SLR between 2000 and 2010. Assuming ice sheet models were capable of describing exactly the ice dynamical conditions in 2000 , and also assuming the parameters forcing enhanced ice discharge to be properly known, we can compute a broad scale of uncertainties on predicted SLR arising from the use of the four different models. If we arbitrarily consider the $F S-A G$ model as the one that would give the SLR prediction of $4.6 \mathrm{~mm}$ in 2010 after a given perturbation, the use of the other models would lead to an erroneous contribution to SLR between $3 \mathrm{~mm}$ (under estimation of $30 \%$ by $S S A-P S M G$ ) and $18 \mathrm{~mm}$ (over estimation of $300 \%$ by $S S A-H-F G$ ). Furthermore, as ice sheets are still in a transient phase (i.e. perturbations are sustained through time), the discrepancy of the models would eventually increase with time integration. Of course, these assertions have to be moderated by the fact that the complexity of actual 3-D geometries could mitigate the discrepancy between model results, which is the focus of future research.

\section{Conclusions}

We have computed the transient response of four flowline ice-sheet models to a reduction in the buttressing force exerted by an ice shelf onto the upstream grounded ice sheet. The intensity of buttressing perturbations was chosen in order to reproduce changes in geometry that are comparable to those observed on current ice sheets. Compared to MISMIP, we investigated the transient response in more detail and applied a perturbation that reflects direct mechanical forcing.

The dynamics (or momentum balance) are implemented in a different way in the different models (from SSA to the solution of the full Stokes equations), while the models differ in their numerical treatment as well (finite difference and finite element). One of the models includes the heuristic rule of Pollard and DeConto (2009), i.e. the flux-thickness relation proposed by Schoof (2007), which is imposed at the GL. All models have successfully participated in the MISMIP benchmark (Pattyn et al., 2012a), exhibiting unique stable positions on downward sloping beds, unstable GL positions on retrograde slopes and related hysteresis behaviour over an undulated bedrock.

Surprisingly, and despite the different physics and numerics implemented, all models broadly give similar results in terms of changes in surface geometry and migration of the GL. However, discrepancies remain in between models. Particularly, the $S S A-H-F G$ model which directly implements the boundary layer theory exhibits faster grounding line retreat and larger surface thinning (Figs. 2 and 3). Once cumulated over years, this leads to significant differences in predicted discharge. Moreover, the prescription of flux at the GL introduces high frequency and large amplitude numerical noise deteriorating the surface change signal over decadal 
time scales. Finally, it seems that, at least in these experiments, the boundary-layer theory overestimates the discharge during the transient evolution. As a consequence, models that prescribe the flux at the GL should be used with particular caution when dealing with small spatial and temporal scales.

Estimation of the contribution to SLR through numerical modelling still exhibits large uncertainties, with results from different models showing $>100 \%$ spread on a decadal timescale and still around $40 \%$ two hundred years after the initial change in buttressing. This indicates there may be a large uncertainty in models that are seeking to establish reliable projection of upcoming contribution of the Antarctic ice sheet to SLR. Further model intercomparisons must be pursued to better constrain the rate of discharge, and intercomparisons on specific Antarctic outlet glaciers should be encouraged in the near future.

\section{Appendix A}

In this appendix, the relation between the buttressing factors $\theta$ in Eq. (17) and $C_{F}$ in Eq. (20) is derived. The ice-shelf equation is

$2 \frac{\partial\left(h \tau_{x x}\right)}{\partial x}=\frac{\gamma}{2} \frac{\partial\left(h^{2}\right)}{\partial x}$,

where $h$ is the ice thickness along the ice shelf. The longitudinal deviatoric stress within the ice shelf is then obtained as follows:

$\tau_{x x}=\frac{\gamma}{4} h-\frac{B}{h}$

where $B$ is the back-force at the calving front. Evaluatting this at $x=x_{\mathrm{f}}$ and using Eq. (20), we obtain the following:

$\left.\tau_{x x}\right|_{x_{\mathrm{f}}}=C_{F} \frac{\gamma}{4} h_{\mathrm{f}}=\frac{\gamma}{4} h_{\mathrm{f}}-\frac{B}{h_{\mathrm{f}}}$,

yielding

$B=\left(1-C_{F}\right) \frac{\gamma}{4} h_{\mathrm{f}}^{2}$,

and

$\tau_{x x}=\frac{\gamma}{4}\left(h-\left(1-C_{F}\right) \frac{h_{\mathrm{f}}^{2}}{h}\right)$.

Now, at the GL $x=x_{\mathrm{g}}$, by definition of $\theta$ Eq. (17), buttressing is now defined as the following:

$\left.\tau_{x x}\right|_{x_{\mathrm{g}}}=\theta \frac{\gamma}{4} h_{\mathrm{g}}$

so that

$\theta=1-\left(1-C_{F}\right)\left(\frac{h_{\mathrm{f}}}{h_{\mathrm{g}}}\right)^{2}$.

\section{Appendix B}

In this appendix, we demonstrate how the buttressing pressure $p_{\mathrm{b}}(t)$ in Eq. (22) is obtained, giving the front-stress for the $F S-A G$ model. We need to find $p_{\mathrm{b}}(t)$ such that the mean longitudinal Cauchy stress be the same for all models. This equality is expressed as follows:

$\bar{\sigma}_{x x}{ }^{\mathrm{SSA}}=\bar{\sigma}_{x x}{ }^{\mathrm{FS}}$,

where $\bar{\sigma}_{x x}$ SSA and $\bar{\sigma}_{x x}$ FS are the longitudinal Cauchy stress of SSA models and the $F S-A G$ model, respectively.

The mean longitudinal Cauchy stress for SSA models reads as follows:

$\bar{\sigma}_{x x} \mathrm{SSA}=2 \bar{\tau}_{x x}+\bar{\sigma}_{z z}$,

where $\bar{\sigma}_{z z}=-\frac{\rho_{i} g h_{\mathrm{f}}}{2}$ and $\bar{\tau}_{x x}$ is given by Eq. (20).

The longitudinal Cauchy stress for $F S-A G$ model, given by Eq. (22), and once integrated over the ice column givesthe following:

$\bar{\sigma}_{x x}{ }^{\mathrm{FS}}=-\frac{\rho_{\mathrm{w}} g z_{\mathrm{b}}^{2}}{2 h_{\mathrm{f}}}+p_{\mathrm{b}}$.

Using Eq. (B2) for SSA models and Eq. (B3) for $F S-A G$, Eq. (B1) leads to the following:

$2 C_{F} \frac{\gamma}{4} h_{\mathrm{f}}-\frac{\rho_{i} g h_{\mathrm{f}}}{2}=-\frac{\rho_{\mathrm{w}} g z_{\mathrm{b}}^{2}}{2 h_{\mathrm{f}}}+p_{\mathrm{b}}$.

Using the flotation condition $\rho_{i} h_{\mathrm{f}}=\rho_{\mathrm{w}} z_{\mathrm{b}}$, and after simplifications, $p_{\mathrm{b}}$ can be isolated and deduced as the following:

$p_{\mathrm{b}}=\frac{\rho_{\mathrm{w}} g z_{\mathrm{b}}^{2}}{2 \rho_{i} h_{\mathrm{f}}}\left(\rho_{\mathrm{w}}-\rho_{i}\right)\left(C_{F}-1\right)$.

Acknowledgements. Some of the computations presented in this paper were performed using the CIMENT infrastructure (https://ciment.ujf-grenoble.fr), which is supported by the RhôneAlpes region (GRANT CPER07_13 CIRA: http://www.ci-ra.org). This work was also supported by funding from the ice2sea programme from the European Union 7th Framework Programme, grant number 226375. Ice2sea contribution number 127.

Edited by: E. Larour

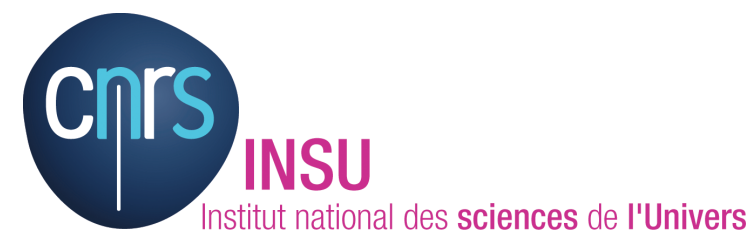

The publication of this article is financed by CNRS-INSU. 


\section{References}

Bindschadler, R. A., Nowicki, S., Abe-Ouchi, A., Aschwanden, A., Choi, H., Fastook, J., Granzow, G., Greve, R., Gutowski, G., Herzfeld, U., Jackson, C., Johnson, J., Khroulev, C., Levermann, A., Lipscomb, W. H., Martin, M., Morlighem, M., Parizek, B. R., Pollard, D., Price, S., Ren, D., Saito, F., Seddik, H., Seroussi, H., Takahashi, K., Walker, R., and Wang, W.: Ice-Sheet Model Sensitivities to Environmental Forcing and Their Use in Projecting Future Sea-Level (The SeaRISE Project), J. Glaciol., accepted, 2012.

Docquier, D., Perichon, L., and Pattyn, F.: Representing Grounding Line Dynamics in Numerical Ice Sheet Models: Recent Advances and Outlook, Surv. Geophysics, 32, 417-435, doi:10.1007/s10712-011-9133-3, 2011.

Durand, G., Gagliardini, O., de Fleurian, B., Zwinger, T., and Le Meur, E.: Marine ice sheet dynamics: Hysteresis and neutral equilibrium, J. Geophys. Res., 114, F03009, doi:10.1029/2008JF001170, 2009a.

Durand, G., Gagliardini, O., Zwinger, T., Le Meur, E., and Hindmarsh, R. C. A.: Full-Stokes modeling of marine ice sheets: influence of the grid size, Ann. Glaciol., 50, 109-1114, 2009b.

Fornberg, B.: A Practical guide to pseudospectral methods, 1996.

Gagliardini, O., Durand, G., Zwinger, T., Hindmarsh, R. C. A., and Le Meur, E.: Coupling of ice-shelf melting and buttressing is a key process in ice-sheets dynamics, Geophys. Res. Lett., 37, L14501, doi:10.1029/2010GL043334, 2010.

Gillet-Chaulet, F. and Durand, G.: Glaciology: Ice sheet Advance in Antarctica, Nature, 467, 794-795, 2010.

Gillet-Chaulet, F., Gagliardini, O., Seddik, H., Nodet, M., Durand, G., Ritz, C., Zwinger, T., Greve, R., and Vaughan, D. G.: Greenland ice sheet contribution to sea-level rise from a new-generation ice-sheet model, The Cryosphere, 6, 1561-1576, doi:10.5194/tc-6-1561-2012, 2012.

Glen, J.: The creep of polycristalline ice, Proc. R. Soc. A., 228, 519-538, 1955.

Hindmarsh, R. C. A.: Qualitative dynamics of marine ice sheets, Ice in the Climate System, edited by: Peltier, W. R., 68-99, 1993.

Hindmarsh, R. C. A.: The role of membrane-like stresses in determining the stability and sensitivity of the Antarctic ice sheets: back-pressure and grounding line motion, Phil. Trans. R. Soc. A, 364, 1733-1767, doi:10.1098/rsta.2006.1797, 2006.

Hindmarsh, R. C. A.: An observationally validated theory of viscous flow dynamics at the ice-shelf calving front, J. Glaciol., 58, 375-387, 2012.

Hindmarsh, R. C. A. and Le Meur, E.: Dynamical processes involved in the retreat of marine ice sheets, J. Glaciol., 47, 271282, 2001.

Larour, E., Schiermeier, J., Rignot, E., Seroussi, H., Morlighem, M., and Paden, J.: Sensitivity analysis of Pine Island Glacier ice flow using ISSM and DAKOTA, J. Geophys. Res., 117, F02009, doi:10.1029/2011JF002146, 2012.

Mac Ayeal, D. R.: Irregular Oscillations of the West Antarctic Ice Sheet, Nature, 365, 214-215, 1992.

MacAyeal, D. R., Hulbe, C. L., Huybrechts, P., Rommelaere, V., Determann, J., and Ritz, C.: An ice-shelf model test based on the Ross ice shelf, Ann. Glaciol., 23, 46-51, 1996.

Morland, L. W.: Unconfined Ice shelf flow, Dynamics of the West Antarctic Ice Sheet, edited by: der Veen, C. J. V. and Oerlemans, J., Cambridge University Press, United Kingdom and New York,
UK, USA, 1987.

Morlighem, M., Rignot, E., Seroussi, H., Larour, E., Dhia, H. B., and Aubry, D.: Spatial patterns of basal drag inferred using control methods from a full-Stokes and simpler models for Pine Island Glacier, West Antarctica, Geophys. Res. Lett., 37, L14502, doi:10.1029/2010GL043853, 2010.

Nick, F. M., Vieli, A., Howat, I. M., and Joughin, I.: Large-scale changes in Greenland outlet glacier dynamics triggered at the terminus, Nat. Geosci., 2, 110-114, doi:10.1029/2005JF000394, 2009.

Pattyn, F., Huyghe, A., Brabander, S. D., and De Smedt, B.: Role of transition zones in marine ice sheet dynamics, J. Geophys. Res., 111, F02004, doi:10.1029/2005JF000, 2006.

Pattyn, F., Schoof, C., Perichon, L., Hindmarsh, R. C. A., Bueler, E., de Fleurian, B., Durand, G., Gagliardini, O., Gladstone, R., Goldberg, D., Gudmundsson, G. H., Huybrechts, P., Lee, V., Nick, F. M., Payne, A. J., Pollard, D., Rybak, O., Saito, F., and Vieli, A.: Results of the Marine Ice Sheet Model Intercomparison Project, MISMIP, The Cryosphere, 6, 573-588, doi:10.5194/tc6-573-2012, 2012.

Pattyn, F., Perichon, L., Durand, G., Favier, L., Gagliardini, O., Hindmarsh, R. C. A., Zwinger, T., Albrecht, T., Cornford, S., Docquier, D., Fuerst, J., Goldberg, D., Gudmundsson, G. H., Humbert, A., Huetten, M., Huybrechts, P., Jouvet, G., Kleiner, T., Larour, E., Martin, D., Morlighem, M., Payne, T., Pollard, D., Rueckamp, M., Rybak, O., Seroussi, H., Thoma, M., , and Wilkens, N.: Grounding-line migration in plan-view marine icesheet models: results of the ice-sea MISMIP3d intercomparison, J. Glaciol., submitted, 2012.

Pollard, D. and DeConto, R. M.: Modelling West Antarctic ice sheet growth and collapse through the past five million years, Nature, 458, 329-333, doi:10.1038/nature07809, 2009.

Pollard, D. and DeConto, R. M.: Description of a hybrid ice sheetshelf model, and application to Antarctica, Geosci. Model Dev., 5, 1273-1295, doi:10.5194/gmd-5-1273-2012, 2012.

Price, S., Payne, A. J., Howat, I. M., and Smith, B. E.: Committed sea-level rise for the next century from Greenland ice sheet dynamics during the past decade, Proc. Nat. Aca of Sci., 108, 8978-8983, 2011.

Pritchard, H. D., Ligtenberg, S. R. M., Fricker, H. A., Vaughan, D. G., Van den Broeke, M., and Padman, L.: Antarctic ice-sheet loss driven by basal melting of ice shelves, Nature, 484, 202-205, 2012.

Rignot, E.: Fast recession of a West Antarctic glacier, Science, 281, 549-551, 1998.

Rignot, E., Bamber, J. L., van den Broeke, M., Davis, C., Li, Y., van de Berg, W. J., and van Meijgaard, E.: Recent Antarctic ice mass loss from radar interferometry and regional climate modelling, Nat. Geosci., 1, 106-110, doi:10.1038/ngeo102, 2008.

Rignot, E., Velicogna, I., van den Broeke, M., Monaghan, A., and Lenaerts, J.: Acceleration of the contribution of the Greenland and Antarctic ice sheets to sea level rise, Geophys. Res. Lett., 38, L05503, doi:10.1029/2011GL046583, 2011.

Schoof, C.: Ice sheet grounding line dynamics: Steady states, stability, and hysteresis, J. Geophys. Res., 112, F03S28, doi:10.1029/2006JF000664, 2007.

Velicogna, I.: Increasing rates of ice mass loss from the Greenland and Antarctic Ice Sheet revealed from GRACE, J. Geophys. Res., 36, L19503, doi:10.1029/2009GL040222, 2009. 
Vieli, A. and Payne, A. J.: Assessing the ability of numerical ice sheet models to simulate grounding line migration, J. Geophys. Res., 110, F01003, doi:10.1029/2004JF000202, 2005.

Weertman, J.: Stability of a junction of an ice sheet and an ice shelf, J. Glaciol., 13, 3-11, 1974.
Williams, C. R., Hindmarsh, R. C. A., and Arthern, R. J.: Frequency response of ice streams, Proc. Roy. Soc. Ser. A., 468, 3285-3310, doi:10.1098/rspa.2012.0180, 2012.

Wingham, D. J., Shepherd, A., Muir, A., and Marshall, J. G.: Mass Balance of the Antarctic Ice Sheet, Phil. Trans. R. Soc. A, 364, 1627-1635, doi:10.1098/rsta.2006.1792, 2006. 